\title{
Analysis of the linearised observation operator in a land surface data assimilation scheme for numerical weather prediction
}

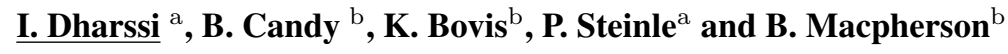 \\ ${ }^{\mathrm{a}}$ The Centre for Australian Weather and Climate Research, Melbourne, Australia \\ ${ }^{\mathrm{b}}$ Met Office, Exeter, United Kingdom \\ Email: I.Dharssi@bom.gov.au
}

\begin{abstract}
Several Meteorological service agencies have developed Extended Kalman Filter based land data assimilation systems that, in principle, can analyse any model land variable. Such systems can make use of a wide variety of observation types, such as screen level ( 2 meters above the surface) observations and satellite based estimates such as retrieved surface soil moisture and retrieved skin temperature. Indirect measurements can be used and information propagated from the surface into the deeper soil layers. A key component of the system is the calculation of the Jacobians of the observation operator which describe the link between the observations and the land surface model variables. The Jacobians are estimated using finite difference by performing short model forecasts with perturbed initial conditions. This paper examines the Jacobians that link observations of screen level variables, satellite derived surface soil moisture and satellite derived skin temperature to model soil temperature and moisture.
\end{abstract}

The calculated Jacobians that link screen level variables to model soil moisture show that there is strong coupling between the screen level and the soil. The coupling between the topmost model level soil moisture and the screen level is found to be due to a number of processes including bare soil evaporation, soil thermal conductivity, soil thermal capacity as well as transpiration by plants. Therefore, there is significant coupling both during the day and at night. The sign of the Jacobians linking screen level temperature to topmost model level soil moisture are usually negative during the day and tends to be positive during the night. The coupling between the screen level and soil moisture in the deeper model layers is primarily through transpiration by plants. Therefore the coupling is only significant during the day and the vertical variation of the coupling is found to be significantly affected by the vegetation root depths. The calculated Jacobians that link screen level temperature to model soil temperature are found to be largest for the topmost model soil layer and become very small for the lower soil layers. These Jacobians are largest during the night and generally positive in value.

It is found that the Jacobians that link observations of surface soil moisture to model soil moisture are strongly affected by the soil hydraulic conductivity. Generally, for the Joint UK Land Environment Simulator (JULES) land surface model, the coupling between the surface and root zone soil moisture is weak. Finally, the Jacobians linking observations of skin temperature to model soil temperature and moisture are calculated. These Jacobians are found to have a similar spatial pattern to the Jacobians for observations of screen level temperature.

Where the linear assumption is valid, the calculated values of the Jacobians should be nearly independent of the sign of the perturbations used. This is investigated by comparing Jacobians calculated using perturbations of opposite signs. Jacobians values that are significantly affected by the sign of perturbation used are assumed to contain a gross error and not used by the data assimilation. A simple quality control scheme is developed to detect land points where the computed Jacobians contain such gross errors. Analysis is also performed of the sensitivity of the calculated Jacobians to the magnitude of the perturbations used.

Keywords: Soil moisture, soil temperature, screen level, land surface, weather prediction 


\section{INTRODUCTION}

Many observational and modelling studies suggest strong coupling between the atmosphere and the land surface. Soil moisture and temperature have a significant impact on screen level temperature and humidity, low clouds and precipitation, by influencing the exchange of heat and moisture between the land surface and the atmosphere (Walker and Rowntree, 1977; Timbal et al., 2002; Dharssi et al., 2009). Soil moisture is important for the prediction of summer-time precipitation at mid-latitudes over land and plays an important role in the development of convective storms (Findell and Eltahir, 1997). The land surface is also very important for the seasonal prediction of extreme events such as heat waves and drought.

Data assimilation (DA) is extremely important for numerical weather prediction (NWP) since errors in the model initial conditions can grow rapidly and seriously degrade forecasts. Specifying the model initial soil moisture and temperature state is especially difficult since there are few near real-time ground based observations of soil moisture and temperature. Therefore, indirect observations are usually used by land surface DA schemes to initialise the model soil moisture and temperature (Dharssi et al., 2011; de Rosnay et al., 2012). To make fuller use of the available global remotely sensed measurements of the land surface as well as screen level observations an Extended Kalman Filter (EKF) based land surface data assimilation system has been developed in collaboration between the Bureau of Meteorology and the Met Office (Dharssi et al., 2012). Such EKF land DA systems can make more statistically optimal use of a wide variety of observation types, such as screen level observations and satellite based estimates such as retrieved Surface Soil Moisture, retrieved skin temperature , Leaf Area Index and Fraction of Photosynthetically Active Radiation (FPAR). Indirect measurements can be used and information propagated from the surface into the deeper soil layers. For example an EKF based land DA system may; a) Use observations of screen level temperature and humidity to analyse soil temperature and moisture. b) Use satellite estimates of surface soil moisture to analyse surface and root-zone soil moisture. c) Use satellite estimates of skin temperature to analyse soil temperature and moisture. d) Use satellite estimates of FPAR to analyse soil moisture.

\section{Land Surface Analysis}

A number of new space-borne remote sensing systems have been developed that provide information on surface soil moisture and temperature. However, most NWP centres still only use screen level observations (of temperature and humidity) for the operational analysis of soil moisture and temperature, e.g. ECMWF (de Rosnay et al., 2012) and Meteo-France (Giard and Bazile, 2000). The Met Office is the first, and thus far only, NWP centre to operationally use satellite derived measurements of surface soil moisture together with screen level observations for the analysis of soil moisture. Dharssi et al. (2011) find that assimilation of remotely sensed surface soil wetness measurements improves the agreement of the soil moisture analyses with ground based soil moisture observations and improves forecasts of screen level temperature and humidity.

\subsection{The Extended Kalman Filter}

The DA problem is kept manageable by using a 1-dimensional approach that assumes that the model land columns are independent of each other. The standard EKF land DA analysis equations for each land column are given by

$$
\begin{aligned}
& \mathbf{x}^{\mathbf{a}}\left(t_{i}\right)=\mathbf{x}^{\mathbf{b}}\left(t_{i}\right)+\mathbf{K}_{i}\left[\mathbf{o}\left(t_{i}\right)-h_{i}\left(\mathbf{x}^{\mathbf{b}}\right)\right] \\
& \mathbf{K}_{i}=\mathbf{B H}_{i}^{T}\left(\mathbf{H}_{i} \mathbf{B} \mathbf{H}_{i}^{T}+\mathbf{R}\right)^{-1} .
\end{aligned}
$$

$\mathbf{x}\left(t_{i}\right)$ represents the state vector of a land column at time $t_{i}$ with superscripts $a$ and $b$ standing for analysis and background. $\mathbf{o}\left(t_{i}\right)$ is the observation vector. $\mathbf{K}_{i}$ is the Kalman gain matrix at time $t_{i}$. $\mathbf{B}$ is the background error covariance matrix. $\mathbf{R}$ is the observation error covariance matrix.

$\mathbf{H}_{i}$ is the Jacobian (linearisation) of the non-linear observation operator $h_{i}$ and is defined using $h_{i}(\mathbf{x}+\boldsymbol{\Delta}) \simeq$ $h_{i}(\mathbf{x})+\mathbf{H}_{i} \boldsymbol{\Delta}$, where $\boldsymbol{\Delta}$ is a small perturbation to the model state $\mathbf{x}$. The elements of $\mathbf{H}_{i}$ are estimated using finite difference by individually perturbing each component of $\mathbf{x}$ by a a small scalar amount $\delta_{j}$. A given element of $\mathbf{H}_{i}$ is calculated using

$$
H_{k j}=\frac{y_{k}\left(\mathbf{x}+\delta_{j}\right)-y_{k}(\mathbf{x})}{\delta_{j}} .
$$


$y_{k}\left(\mathbf{x}+\delta_{j}\right)$ is a short model forecast of observation type $k$ (e.g. screen level temperature) starting from perturbed initial conditions $\mathbf{x}+\delta_{j}$. The number of perturbed forecasts required increases with the number of model variables to be analysed and the number of soil layers.

The major computational cost of the EKF land DA system is the cost of running the perturbed forecasts. ECMWF use the fully coupled land/atmosphere model for the perturbed forecasts. Meteo France use an offline land surface model (uncoupled to the atmosphere model) for the perturbed forecasts. Consequently the Meteo France EKF land DA system is computationally several orders of magnitude cheaper. Our EKF land DA system also uses an off-line land surface model for the perturbation forecasts. Balsamo et al. (2007) and Mahfouf et al. (2009) have shown that the off line land surface model can be used to reliably calculate $\mathbf{H}_{i}$. Atmospheric forcing of air temperature and humidity are applied at a height of $20 \mathrm{~m}$ (which is above the screen level). This allows the EKF land DA system to also assimilate observations of screen level temperature and humidity (see Fig. 1 of Mahfouf et al. (2009) for a fuller explanation).

\subsection{The Land Surface Model}

The ACCESS (Australian Community Climate and Earth-System Simulator) system is used at the Bureau for NWP. Our EKF land DA system uses JULES (Best et al., 2011) to represent the land surface processes. The soil is discretised into four layers of $0.1,0.25,0.65$ and $2 \mathrm{~m}$ thickness (from top to bottom). The vertical discretisation for the soil hydrology is the same as that for the thermodynamics. The soil hydrology is based on a finite difference form of the Richards equation and Darcy's law. The van Genuchten equations are used to describe the relationship of soil hydraulic conductivity and soil suction to the unfrozen volumetric soil moisture. The soil thermodynamics is represented by diffusive heat exchanges between the soil layers and by heat advection between the soil layers by the fluxes of moisture. The freezing and melting of soil water are also represented and the associated latent heat is included in the thermodynamic calculations. Transpiration by plants extracts soil water directly from the soil layers via the plant roots while bare soil evaporation extracts soil water from the top soil layer only. The ability of plants to access water from each soil layer is determined by the root density distribution and soil moisture availability.

\section{Results ANd CONCLUSions}

The most important aspect of the EKF land data assimilation system is the calculation of the Jacobians of the observation operator that describe the link between the observations and the land surface model variables. Other works such as Mahfouf et al. (2009) and Drusch et al. (2009) have also looked at the calculation of the Jacobians. However, this work examines the Jacobians in much greater detail than before. In addition, this is the first work to use the JULES land surface model to compute the Jacobians for screen level observations and measurements of surface skin temperature.

Figure 1 shows an example of the computed Jacobians of screen level temperature with respect to soil moisture in the four model soil layers. For soil layers two to four, the coupling between screen level temperature and soil moisture is primarily through transpiration by vegetation. Consequently the coupling occurs during daylight. Negative Jacobians values means that an increase in soil moisture leads to a cooling of the screen level. For the surface soil layer, the picture is more complicated as there is strong coupling both during the day and at night. The Jacobians have a positive value in some places and a negative value in others.

Results show that quality control of the computed Jacobians is very important. Two quality control schemes are compared; a computationally expensive scheme that doubles the number of perturbed forecasts required and a simple quality control scheme that is computationally cheap. The use of the simple quality control scheme is found to give adequate results. Results also show that the computed Jacobians can be sensitive to the size of the perturbations used. Perturbations that are too small cause problems due to numerical rounding while perturbations that are too large cause problems due to non-linearities in the model. Experiments show that volumetric soil moisture perturbation values in the range of $10^{-4}$ to $10^{-2} \mathrm{~m}^{3} / \mathrm{m}^{3}$ give good results and a perturbation value of $10^{-3} \mathrm{~m}^{3} / \mathrm{m}^{3}$ is close to optimal (see Figure 2). For skin and soil temperature perturbations, experiments indicate that a perturbation value of $10^{-1} \mathrm{~K}$ is close to optimal.

This is the first work to look at the effect of land surface parameterisations on the computed Jacobians. As expected, the parameterisation details have a significant impact. Experiments are performed where the parameterisations are modified or switched off. Results show that the coupling between the soil moisture in the topmost model layer and the screen level is due to a number of processes including bare soil evaporation, soil thermal conductivity, soil thermal capacity as well as transpiration by plants. The coupling between the soil 

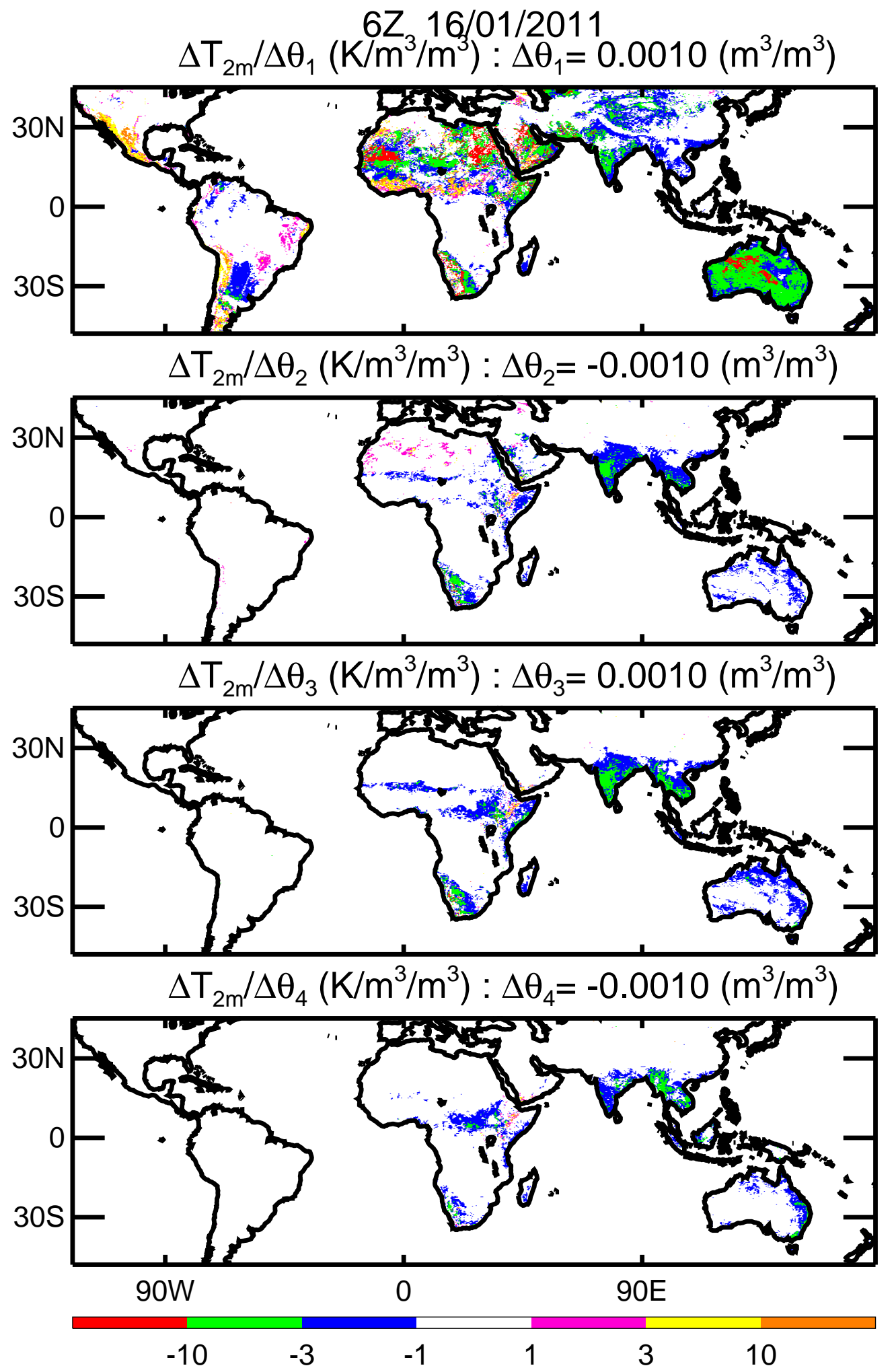

Figure 1. Example of the computed Jacobians of screen level temperature with respect to volumetric soil moisture in the four model soil layers. The Jacobians are valid at 6Z 16/01/2011 and are calculated using three hour long perturbed forecasts with initial soil moisture perturbations of magnitude $\left|\Delta \theta_{l}\right|=10^{-3} \mathrm{~m}^{3} / \mathrm{m}^{3}$. The sign of $\Delta \theta_{l}$ can be positive or negative and for illustration is alternated between soil layers. 

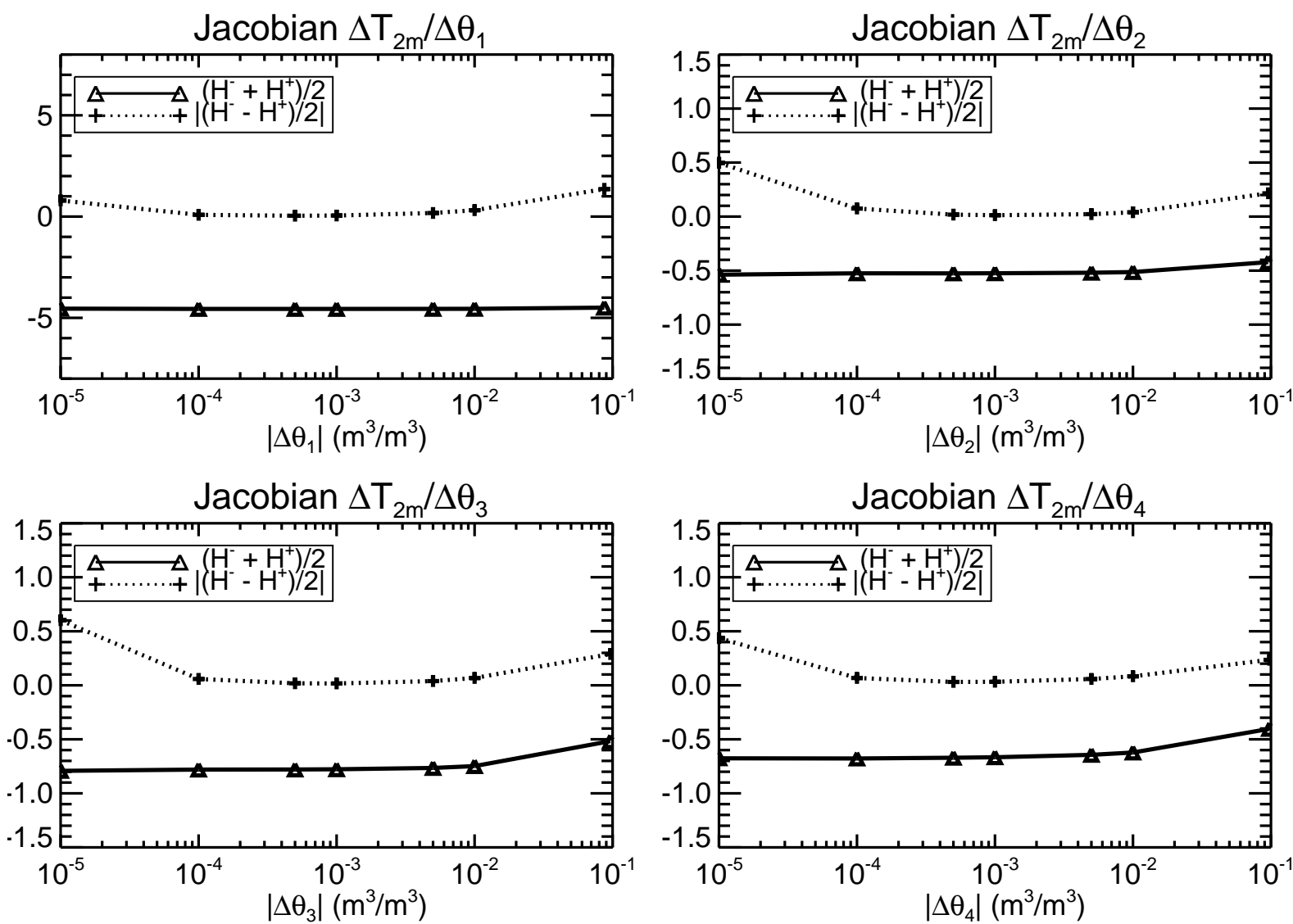

Figure 2. The sensitivity of the calculated Jacobians for screen level temperature to the magnitude of the volumetric soil moisture perturbations $\left|\Delta \theta_{l}\right|$ in soil layer $l$. $H^{-}$represents Jacobian values calculated using a negative perturbation while $H^{+}$represents Jacobian values calculated using a positive perturbation. For a well behaved EKF system $\left|H^{-}-H^{+}\right|$should be close to zero. The dotted line shows $\left|H^{-}-H^{+}\right| / 2$ averaged over the Australia region while the solid line shows $\left(H^{-}+H^{+}\right) / 2$ averaged over Australia. The results indicates that the system is well behaved for $\left|\Delta \theta_{l}\right|$ values in the range of $10^{-4}$ to $10^{-2} \mathrm{~m}^{3} / \mathrm{m}^{3}$.

moisture in the lower model layers and the screen level is due to transpiration by plants. This result is significant as it explains why the coupling with the soil moisture in the topmost model layer is much stronger than the coupling with the soil moisture in the lower model layers. Consequently, soil moisture increments in the topmost model layer will be larger than would be the case if the coupling were only due to transpiration. In addition, improving the analysis of topmost model layer soil moisture will have a significant impact on forecasts of screen level temperature and humidity. The Jacobians linking observations of surface soil moisture with soil moisture in the lower model layers have been computed. Experiments show that artificially increasing the soil hydraulic conductivity by a factor of ten significantly increases the coupling between the surface and root zone soil moisture (Figure 3). Otherwise, for JULES, the coupling between the surface and root zone soil moisture is weak. The van Genuchten (1980) equations describe the model relationship between the soil hydraulic conductivity $\left(K_{V G}\right)$ and the unfrozen volumetric soil moisture $\theta^{u}$.

$$
K_{V G}=K_{s} S_{e}^{L}\left[1-\left(1-S_{e}^{1 / m}\right)^{m}\right]^{2},
$$

where the soil wetness $S_{e}=\left(\theta^{u}-\theta_{r}\right) /\left(\theta_{s}-\theta_{r}\right), L=0.5$ and $m=1-1 / n . \theta_{s}, \theta_{r}, K_{s}, \alpha$ and $n$ are the van Genuchten soil parameters and depend on the soil texture (size distribution of the soil particles and the soil organic carbon content). Equation 4 shows that the hydraulic conductivity is very sensitive to changes in soil moisture. Small changes in soil moisture can lead to order of magnitude changes in soil hydraulic conductivity. For example, when $S_{e}=1, K_{V G}=K_{s}$ while when $S_{e}=0.9$ and $n=1.18, K_{V G}=K_{s} \times 10^{-2}$. In addition, $K_{s}$ is strongly affected by soil texture. For coarse textured soils the model assumes $K_{s}=1.95 \times 10^{-2} \mathrm{~mm} / \mathrm{s}$ while for medium textured soils $K_{s}=2.8 \times 10^{-3} \mathrm{~mm} / \mathrm{s}$. Consequently, uncertainty in soil texture can 


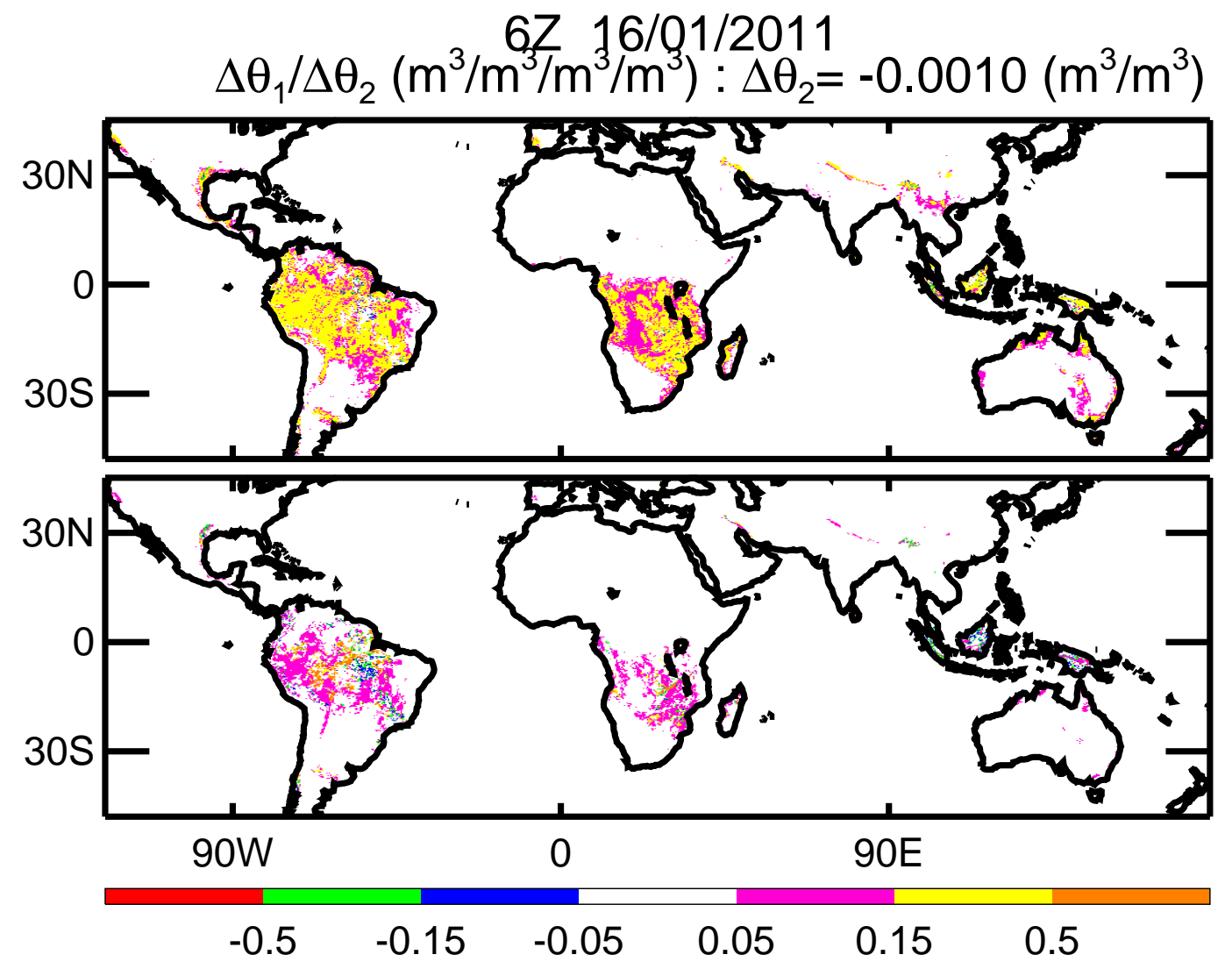

Figure 3. Examples of the computed Jacobians of surface volumetric soil moisture with respect to volumetric soil moisture in the second model soil layer. The upper panel shows the computed Jacobians when the model soil hydraulic conductivity is increases by a factor of ten. The lower panel shows the computed Jacobians when the standard model soil hydraulic conductivity is used.

also lead to order of magnitude uncertainty in soil hydraulic conductivity. Real soils often show significant vertical variation of texture, with surface soils tending to be coarser (larger soil particles) than the sub-surface soils. Therefore, we would expect real soils to usually show significantly higher values of the soil saturated hydraulic conductivity, $K_{s}$, near the surface. Rooney and Claxton (2006) find that increasing $K_{s}$ near the surface improves the ability of a land surface model to simulate the correct soil moisture behaviour. However, most studies (including ours) ignore any vertical variation of soil texture and this most likely explains the weak coupling between the model surface and sub-surface soil moisture.

Using four different land surface models with different coupling strengths and synthetic observations of surface soil moisture, Kumar et al. (2009) find that the potential of surface soil moisture assimilation to improve root zone soil moisture is higher when the coupling between the surface soil moisture and root zone soil moisture is stronger. Given that the true strength of coupling between the surface soil moisture and root zone soil moisture is unknown, the non-identical twin, assimilation experiments of Kumar et al. (2009) suggest that it is better to over-estimate rather than under-estimate the coupling between the surface soil moisture and root zone soil moisture. Therefore, artificially increasing the model surface soil hydraulic conductivity by a factor of ten may be an effective technique to improve the assimilation of satellite derived surface soil moisture. However, careful and comprehensive testing will be required to fully validate all the consequences of such an approach.

The Jacobians linking observations of skin temperature to model soil temperature and moisture have also been computed. These Jacobians have a similar spatial pattern to the Jacobians linking observations of screen level temperature to model soil temperature and moisture but are larger in magnitude. Consequently, assimilation of satellite derived skin temperature may also significantly improve the analysis of model soil temperature and moisture. 


\subsection{Operational Implementation}

The new EKF land surface DA scheme is now used operationally at the UK Met Office in their weather forecasting system to analyse soil moisture using screen level observations and satellite derived surface soil wetness measurements from the Advanced Scatterometer (ASCAT).

\section{REFERENCES}

Balsamo, G., J. Mahfouf, S. Belair, and G. Deblonde, 2007: A land data assimilation system for soil moisture and temperature: an information content study. Journal of Hydrometeorology, 8, 1225-1242.

Best, M. J., M. Pryor, D. B. Clark, G. G. Rooney, R. L. H. Essery, C. B. Ménard, J. M. Edwards, M. A. Hendry, A. Porson, N. Gedney, L. M. Mercado, S. Sitch, E. Blyth, O. Boucher, P. M. Cox, C. S. B. Grimmond, and R. J. Harding, 2011: The Joint UK Land Environment Simulator (JULES), model description - Part 1: Energy and water fluxes. Geoscientific Model Development, 4, 677-699, doi:10.5194/gmd-4-677-2011.

de Rosnay, P., M. Drusch, D. Vasiljevic, G. Balsamo, C. Albergel, and L. Isaksen, 2012: A simplified Extended Kalman Filter for the global operational soil moisture analysis at ECMWF. Quarterly Journal of the Royal Meteorological Society, n/a-n/a, doi:10.1002/qj.2023.

Dharssi, I., K. J. Bovis, B. Macpherson, and C. P. Jones, 2011: Operational assimilation of ASCAT surface soil wetness at the Met Office. Hydrology and Earth System Sciences, 15, 2729-2746, doi:10.5194/hess-152729-2011.

Dharssi, I., P. Steinle, and B. Candy, 2012: Towards a Kalman Filter based land surface data assimilation scheme for ACCESS. CAWCR Technical Report 54, The Centre for Australian Weather and Climate Research, Melbourne, Australia.

URL http: //www. cawcr.gov.au/publications/technicalreports/CTR_054.pdf

Dharssi, I., P. Vidale, A. Verhoef, B. Macpherson, C. Jones, and M. Best, 2009: New soil physical properties implemented in the Unified Model at PS18. Meteorology Research and Development Technical Report 528, Met. Office, Exeter, UK.

URL http: / / tinyurl.com/UKMOreport528-pdf

Drusch, M., K. Scipal, P. De Rosnay, G. Balsamo, E. Andersson, P. Bougeault, and P. Viterbo, 2009: Towards a Kalman Filter based soil moisture analysis system for the operational ECMWF Integrated Forecast System. Geophys. Res. Lett, 36, L10401.

Findell, K. and E. Eltahir, 1997: An analysis of the soil moisture-rainfall feedback, based on direct observations from Illinois. Water Resources Research, 33, 725-735.

Giard, D. and E. Bazile, 2000: Implementation of a new assimilation scheme for soil and surface variables in a global nwp model. Monthly Weather Review, 128, 997-1015.

Kumar, S. V., R. H. Reichle, R. D. Koster, W. T. Crow, and C. D. Peters-Lidard, 2009: Role of subsurface physics in the assimilation of surface soil moisture observations. Journal of Hydrometeorology, 10, 15341547, doi:10.1175/2009JHM1134.1.

Mahfouf, J., K. Bergaoui, C. Draper, F. Bouyssel, F. Taillefer, and L. Taseva, 2009: A comparison of two off-line soil analysis schemes for assimilation of screen level observations. J. Geophys. Res, 114, D08105.

Rooney, G. and B. Claxton, 2006: Comparison of the Met Office's surface exchange scheme, MOSES, against field observations. Quarterly Journal of the Royal Meteorological Society, 132, 425-446, doi:10.1256/qj.04.95.

Timbal, B., S. Power, R. Colman, J. Viviand, and S. Lirola, 2002: Does soil moisture influence climate variability and predictability over Australia? Journal of Climate, 15, 1230-1238.

van Genuchten, M., 1980: A closed-form equation for predicting the hydraulic conductivity of unsaturated soils. Soil Sci. Soc. Am. J, 44, 892-898.

Walker, J. and P. R. Rowntree, 1977: The effect of soil moisture on circulation and rainfall in a tropical model. Quarterly Journal of the Royal Meteorological Society, 103, 29-46, doi:10.1002/qj.49710343503. 